Engineering Failure Analysis, Volume 101, July 2019, Pages 257-273

DOI:10.1016/j.engfailanal.2019.03.008

\title{
Analyzing the failure and damage of FRP composite laminates under high strain rates considering visco-plasticity
}

\author{
Sina Eskandari ${ }^{\mathrm{a}, *}$, Francisco M. Andrade Pires ${ }^{\mathrm{b}}$, Pedro P. Camanho ${ }^{\mathrm{b}}$, Hao \\ $\mathrm{Cui}^{\mathrm{c}}$, Nik Petrinic ${ }^{\mathrm{a}}$, Antonio T. Marques ${ }^{\mathrm{b}}$ \\ ${ }^{a}$ Department of Engineering Science, University of Oxford, Parks Road, Oxford, OX1 3PJ, \\ $U K$ \\ ${ }^{b}$ Faculdade de Engenharia, Universidade do Porto, Rua Doutor Roberto Frias, 4200-465 \\ Porto, Portugal \\ ${ }^{c}$ School of Aerospace, Transport and Manufacturing, Cranfield University, Cranfield, UK
}

\begin{abstract}
In order to analyze composite structures under dynamic condition or model manufacturing processes in which high strain rates exist, it is important to develop a constitutive model that regards these effects. In this work, a phenomenological approach is used to formulate a continuum damage for composites in presence of high strain rates. Visco-plastic behavior of the composite is taken into account and the deformation of composite structures under dynamic conditions is studied. Effects of dynamic conditions on the material properties including strength and fracture toughness are included in the modeling. The model can capture the effects of strain rate on material behavior and has a good capability in the prediction of the response and final strength of composite laminates in comparison with the experimental data under medium and high rates.
\end{abstract}

Keywords: Visco-plasticity, Continuum damage model, High strain rate, Failure strength, LaRC failure criteria

\footnotetext{
* Corresponding author

Email address: sina.eskandari@eng.ox.ac.uk (Sina Eskandari)
} 


\section{Introduction}

Impact loading influences the product design in different sectors such as transportation, defence, medical, and consumer goods. Application of composite materials is also growing in these industries. In order to design structures using composite materials which are under dynamic loads, it is necessary to develop constitutive models that account for different mechanisms involved at higher strain rates in order to predict damage and failure. Having constitutive models also provides the possibility of reducing the test costs considerably using simulation. Manufacturing processes of composites like composite machining also need the analysis of materials under high strain rates. This facilitates the creation of digital twins for production units in the context of industry 4.0 to facilitate digital transformation and building smart factories. In this work a comprehensive model is proposed that includes different phenomena taking place during the dynamic loading of fiber reinforced composite polymers and the response of composite structure under them is analyzed. The model includes visco-elasticity, fiber rotation, damage, and visco-plasticity. Having all of them provides a good framework to study the interaction between different ongoing material mechanisms and predict final failure and structural collapse of laminates along with the possibility to observe dominant damage modes, development of plasticity and fracture plane.

Under sensible strain rates, structures usually exhibit different behavior when compared to low strain rate. The type of activated mechanisms depends on the material type. It has been reported in several works that strain rate influences composite behavior and the degree of nonlinearity [1], [2], [3], 4], [5], [6. According to those experimental observations, for strain rates higher than $0.001 S^{-1}$ the effects of dynamic conditions should be considered. The level of sensitivity depends on material constituents and different sets of behavior have reported for different material. For example, glass fiber has shown strain rate dependency [7] while carbon fibers are not rate dependent [6]. On the other hand, most of polymer matrix materials like epoxy have shown high level of 
strain rate sensitivity[? ]. In this paper, carbon fiber polymer composites are taken anto account and the level of rate sensitivity is reflected in model parameters. Researchers have focused on different aspects of high strain rate in different studies. One of those effects is the impact influence on linear elastic properties of composites. Koerber [6] has reviewed strain rate effect on elastic properties of composites materials. Zhou [8] observed no effect under high strain rate on fibers in fiber reinforced composites while several researchers [9, [10, [11, have shown rate dependency for compressive, tensile and shear properties in polymer composites.

Another aspect is plastic behavior of the matrix under high rate that shows nonlinear visco-plasticity. Volger et al. 12 proposed a fully 3D transversely isotropic visco-plastic model for polymer composites and reported a good agreement between numerical model and experimental data for IM7-8552 carbonepoxy composite. This model is based on a model previously introduced for the plastic behavior of polymer composites [13] . Erice et al. 14] compared 3 different approaches to analyze plasticity under rate dependency. The first approach is to incorporate rate dependency just for in-plane shear plasticity, the second one consists in using 3D extension of the plane stress Hill48 anisotropic plastic surface and third is a quadratic yield function inspired by Pucks fracture initiation criterion. The authors reported a good agreement between numerical and experimental analysis in low, medium and high rate for the 2 latter approaches.

In order to capture nonlinear effects of visco-plasticity on failure and final structural collapse, and achieve a comprehensive model, it is important to couple visco-plasticity with the progressive damage model. Aboudi [15] proposed a 3-dimensional continuum theory for fiber-reinforced elastic-viscoplastic composites. In this model both fiber and matrix are assumed to be elastic-viscoplastic. The author also included work hardening in the model. Weeks et al. 1] modeled nonlinear behavior of composites under strain rate. They have used one parameter plastic potential function to analyze rate dependency of the material. It is assumed that the material is linear elastic in fiber direction and is transversely isotropic. They compared the prediction of the model against experimental re- 


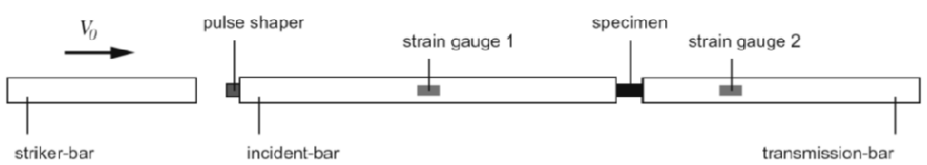

Figure 1: Split-Hopkinson pressure bar setup for dynamic tests [21].

sults for AS4-PEEK which reveal, in some cases, good results though there is still room for improvement. Saleeb [16] analyzed the anisotropic viscoplasticdamage response of composite laminates. Lissenden [17] implemented numerical modelling of damage development with visco-plasticity in metal matrix composites, where the nonlinearity is attributed to the visco-plasticity of the metallic matrix and the inter-facial damage between fibre and matrix. Viscoplasticity is modelled by inclusion of rate dependency in classical plastic theory. Non-linear lamination theory provides the link between the micro and macrolevel in the study. Vasiukov et al. 18 developed a visco-elastic visco-plastic model with anisotropic continous damage model. The visco-plasticity model is based on the modified Hoffman criterion with combination of the Perzyna [19] model. Anisotropic damage model is based on extended three-dimensional damage model. The failure prediction of the T300/1034-C carbon/epoxy laminate with central hole under tension was investigated and showed a good accuracy.

Kuhn et al. [20], 21] have found that the fracture toughness increases under dynamic loads. They have conducted an experimental study to measure compressive crack closure resistance of unidirectional laminates IM7-8552 under strain rates $100 S^{-1}$. The dynamic tests are executed with the split-Hopkinson pressure bar that is shown schematically in Fig.1.

A considerable body of research has been conducted on composite materials under high strain rate in the scope of micro-mechanical analysis [22, [23, [24], [25, [26]. Megnis \& Varna 24] analyzed inelastic behavior of composite materials by Schaperys nonlinear visco-elastic, visco-plastic material model. The visco-elastic behavior is regarded linear and visco-plasticity is assumed nonlinear. Micromechanical Hashins Concentric Cylinder Assembly model is used in 
the visco-elastic formulation to calculate the coefficients of linear visco-elastic compliance functions of UD composite. However, micro-mechanical analysis is computationally expensive and difficult to use at the structural level. In this work a meso-scale model is used to study composite materials which offers a balance between accuracy and cost.

Al-Haik et al. 27] compared explicit and implicit visco-plastic models for carbon-fiber/polymer matrix composites. In the implicit model, the experimental data is used to train artificial neural networks with truncated Newton algorithm, and in the explicit approach, the model which described earlier [1] was utilized. Temperature-dependent material constants are also applied.

In this work a comprehensive constitutive model at the meso-scale is introduced that considers visco-elasticity and visco-plasticity and couples it with continuum damage. The model will be validated by experimental data in medium and high strain rates.

\section{Constitutive model}

In previous work of the authurs [28, [29, 30] a constitutive model was developed that considers elastic behavior, damage, and fiber rotation phenomena at the meso-scale for composite laminates. Choosing a failure criteria is a difficult task since there is no criteria that can accurately predict the failure for all of loading scenarios. The failure model selected is LaRC failure criteria 31] which uses a phenomenological approach and determines the failure mode. After reaching any failure mode, the corresponding damage parameter is triggered. The introduced damage parameters are $d_{1}, d_{2}, d_{6}$, representing damage in the fiber, matrix and shear respectively. Damage initiation and evolution are calculated based on Maimi continuum damage model [32, 33] which is based on stiffness degradation of lamina by applying relevant damage parameter to stiffness component. During the loading, the fiber can rotate and change the initial material direction from laminae $(\boldsymbol{X})$ to the real material direction $(\boldsymbol{x})$ in terms of continuum mechanics. The real material direction is also calculated 
and used based on previous work on fiber rotation 28. In order to avoid repetition, the details of the formulation of those models are not brought here and readers can refer to the cited papers for full details.

In this work, the dynamic effects will be incorporated in that model. In order to do so, 3 mechanisms should be taken into account in addition to discussed ones. Firstly, visco-elasticity should be regarded. The elastic properties, and strengths of material will be affected under dynamic conditions. Secondly, viscoplasticity will be involved. The visco-plastic is produced after matrix yielding and under high strain rates. A 3-dimensional orthotropic visco-plastic model is utilized for modeling of this mechanism. Finally, fracture energy is also influenced by dynamic loads. Therefore, it should be updated based on dynamic values for high strain rates. The constitutive model in real material coordinate system $(\boldsymbol{x})$ is considered as:

$$
\boldsymbol{\sigma}^{\boldsymbol{x}}=\boldsymbol{K}_{d}^{\boldsymbol{x} d y}\left(\boldsymbol{\epsilon}^{\boldsymbol{x}}-\boldsymbol{\epsilon}_{v p}^{\boldsymbol{x}}\right) .
$$

Where, $\boldsymbol{K}_{d}^{\boldsymbol{x} d y}$ is the damaged stiffness under dynamic loads in the real material orientation, where "dy" represents dynamic conditions, " $d$ " represents damaged material, and "x" real material direction. The symbol $\boldsymbol{\epsilon}_{v p}^{\boldsymbol{x}}$ is visco-plastic strain tensor, and $\boldsymbol{\epsilon}^{x}$ is total strain tensor in the real material coordinate system. The procedure to transform stress and strain from the real material direction $(\boldsymbol{x})$ to the initial material direction $(\boldsymbol{X})$ and vice versa is based on the calculated fiber rotation, were discussed in [28] and [29]. The damaged stiffness under quasi-static loads, $\boldsymbol{K}_{d}^{\boldsymbol{x}}$, was also introduced. In order to find stiffness under dynamic loads, the elastic properties and final strength of material should be updated based on strain rate. Indeed, the dynamic stiffness is a function of the quasi-static stiffness as:

$$
\boldsymbol{K}_{d}^{\boldsymbol{x} d y}=\boldsymbol{K}_{d}^{\boldsymbol{x}}\left(E_{i j}^{d y}, X_{i j}^{d y}\right),
$$

where, $E_{i j}^{d y}$ represents the elastic properties and $X_{i j}^{d y}$ the strengths under dynamic loads. Koerber [6] conducted a research to identify the relation of ma- 
terial properties between dynamic and quasi-static modes. The chosen material in that research is carbon/epoxy IM7-8552. According to those analysis $E_{22}$, $E_{33}, G_{12}, G_{13}, G_{23}, X_{C}, Y_{T}, Y_{C}$, and $S_{L}$ are sensitive to strain rate in carbon/epoxy IM7-8552. The relation for a property in quasi-static and dynamic modes which is presented by Koerber [6] based on experimental analysis reads:

$$
E_{i j}^{d y}=E_{i j} f(\dot{\epsilon})
$$

where, $h(\dot{\epsilon})$ is:

$$
f(\dot{\epsilon})=1+(m \dot{\epsilon})^{\frac{1}{n}},
$$

where, $m$ and $n$ are experimental factors that are defined based on experiments and the fact that if the property is for elastic or strength behavior, as summarized for IM7-8552 in Table 1.

Table 1: Experimental factor for defining IM7-8552 properties in dynamic model [6].

\begin{tabular}{ccc}
\hline Property type & $m$ & $n$ \\
\hline Elastic & $1.60 \times 10^{-4}$ & 2 \\
Strength & $1.13 \times 10^{-4}$ & 4 \\
\hline
\end{tabular}

Elastic and strength properties of the material are brought in Tables 2 and 3. respectively.

Table 2: IM7-8552 elastic properties 34.

\begin{tabular}{cccc}
\hline$E_{1}(\mathrm{GPa})$ & $E_{2}(\mathrm{GPa})$ & $G_{12}(\mathrm{GPa})$ & $\nu_{12}$ \\
\hline 150 & 11 & 4.6 & 0.3 \\
\hline
\end{tabular}

Some strength properties of composite materials may change based on the thickness and position of the ply in configuration of lamina. LaRC failure criteria 
Table 3: IM7-8552 unidirectional strengths (MPa) 34.

\begin{tabular}{ccccc}
\hline$X_{T}$ & $X_{C}$ & $Y_{T}$ & $Y_{C}$ & $S_{L}$ \\
\hline 2323.5 & 1200.1 & 62.3 & 199.8 & 92.3 \\
\hline
\end{tabular}

takes this effect into account. In this research in-situ properties of laminae is regarded based on formulations proposed by [35]. The In-situ properties of IM7-8552 are presented in Table 4 .

Table 4: In situ strengths (MPa).

\begin{tabular}{cccc}
\hline & Embedded ply & Outer ply & Thick ply \\
\hline$Y_{T}$ & 160.2 & 101.4 & 113.3 \\
$S_{L}$ & 130.2 & 106.9 & 106.9 \\
\hline
\end{tabular}

By defining Eq.(4), material properties which are sensitive to strain rate can be updated, and based on them, the stiffness and damage variables will be defined. Therefore, it is necessary to calculate visco-plastic strain, $\boldsymbol{\epsilon}_{v p}^{x}$.

In order to define the visco-plastic strain a non associative flow rule is used. Therefore, it is necessary to define yield, $f$, and plastic potential, $g$, functions. These function and the procedure to calculate visco-plastic strain is followed according to the method proposed by [12 that is an extension to [13] . By having $f$ and $g$, the visco-plastic strain will be defined by:

$$
\dot{\boldsymbol{\epsilon}}_{v p}=\gamma_{n+1}^{v p} \boldsymbol{n}_{g}=\frac{\left\langle f\left(\boldsymbol{\sigma}, \bar{\epsilon}_{v p}, \boldsymbol{A}\right)\right\rangle^{m}}{\eta} \boldsymbol{n}_{g},
$$

where, $\eta$ and $m$ are visco-plastic parameters and $A$ is structural tensor. In developing the yield surface, it is assumed that the stiffness matrix is not symmetric and the anisotropy is incorporated by so called structural tensor that is dyadic product of preferred direction, $\mathbf{a}$, that is the fiber direction in 
unidirectional fiber composites, as:

$$
A=\boldsymbol{a} \otimes \boldsymbol{a}
$$

Since the rest of equations lead to a symmetric stiffness and expressed in the material coordinate system, the visco-plastic strain that is computed using this procedure should be rotated to be compatible with the rest of the equations. The input of this computations should also be in global coordinate system. The quantity $\boldsymbol{n}_{g}$ is the flow direction and defined by:

$$
\boldsymbol{n}_{g}=\frac{\partial g(\boldsymbol{\sigma}, \boldsymbol{A})}{\partial \boldsymbol{\sigma}},
$$

Yield function and plastic function are defined by Volger [12] based on defined invariants as:

$$
\boldsymbol{f}\left(\boldsymbol{\sigma}, \bar{\epsilon}_{v p}, \boldsymbol{A}\right)=\alpha_{1} I_{1}+\alpha_{2} I_{2}+\alpha_{3} I_{3}+\alpha_{32} I_{3}^{2}-1 \leq 0,
$$

and,

$$
\boldsymbol{g}(\boldsymbol{\sigma}, \boldsymbol{A})=\beta_{1} I_{1}+\beta_{2} I_{2}+\beta_{3} I_{3}^{2}-1 .
$$

The scalers $\alpha_{i}$ and $\beta_{i}$ will be defined according to experimental tests for the material which is being studied. The utilized invariants read:

$$
\begin{gathered}
I_{1}=\frac{1}{2} \operatorname{tr}\left(\boldsymbol{\sigma}^{p i n d}\right)^{2}-\boldsymbol{a}\left(\boldsymbol{\sigma}^{p i n d}\right)^{2} \boldsymbol{a}, \\
I_{2}=\boldsymbol{a}\left(\boldsymbol{\sigma}^{p i n d}\right)^{2} \boldsymbol{a}, \\
I_{3}=\operatorname{tr}(\boldsymbol{\sigma})-\boldsymbol{a}(\boldsymbol{\sigma}) \boldsymbol{a} .
\end{gathered}
$$

Where, $\boldsymbol{\sigma}^{\text {pind }}$ is:

$$
\boldsymbol{\sigma}^{p i n d}=\boldsymbol{\sigma}-\frac{1}{2}(\operatorname{tr}(\boldsymbol{\sigma})-\boldsymbol{a}(\boldsymbol{\sigma}) \boldsymbol{a}) \mathbf{1}+\frac{1}{2}(\operatorname{tr}(\boldsymbol{\sigma})-3 \boldsymbol{a}(\boldsymbol{\sigma}) \boldsymbol{a}) \boldsymbol{A}
$$


The method for finding the visco-plastic properties and to calibrate them is discussed in [12].

The fracture toughness is $165.6 \frac{K J}{m^{2}}$ when it is subjected to dynamic loads according to the study of [20. This updated fracture energy is used in this work for longitudinal fracture energy in compression and the rest of fracture toughness values are considered according to quasi static ones from [33]. The utilized values of fracture toughness are summarized in Table 5

Table 5: Intralaminar fracture toughness for IM7-8552( $\left.\frac{K J}{m^{2}}\right)[33$.

\begin{tabular}{ccccc}
\hline$G_{1}^{+}$ & $G_{1}^{-}$ & $G_{2}^{+}$ & $G_{2}^{-}$ & $G_{6}$ \\
\hline 89.83 & 165.5 & 0.23 & 0.76 & 0.46 \\
\hline
\end{tabular}

\section{Finite element implementation}

In order to implement the model that includes strain rate effects and viscoplasticity, the VUMAT subroutine is developed in the commercial ABAQUS software. Visco-plasticity implementation is pursued based on research done by [12] that solves the nonlinear equations introduced in previous section(Eq. (5)Eq. (13)). It is necessary to update the consistency parameter, $\Delta \gamma^{v p}$, that is done using consistent linearization. The root of the linearized residual is:

$$
\Delta^{2} \gamma_{n+1}^{(k+1)}=-\frac{f_{n+1}^{(k)}{ }^{m}-\Delta \gamma_{n}^{(k+1)} \frac{\eta}{\Delta t}}{m \cdot f_{n+1}^{(k)^{m-1}}-\Delta \gamma_{n+1}^{(k)} \frac{\eta}{\Delta t}} .
$$

The continuum damage model uses fracture toughness properties of the material that are shown in Table 5 for quasi-static conditions. A set of experimental tests conducted by [5], 6] for unidirectional composite laminates under dynamic loads using Split-Hopskin pressure bar test. The numerical examples were developed to compare the results of the model with experimental results in order to validate the results. The numerical model is executed under medium strain rate in compression for unidirectional laminates of different fiber orientations. 


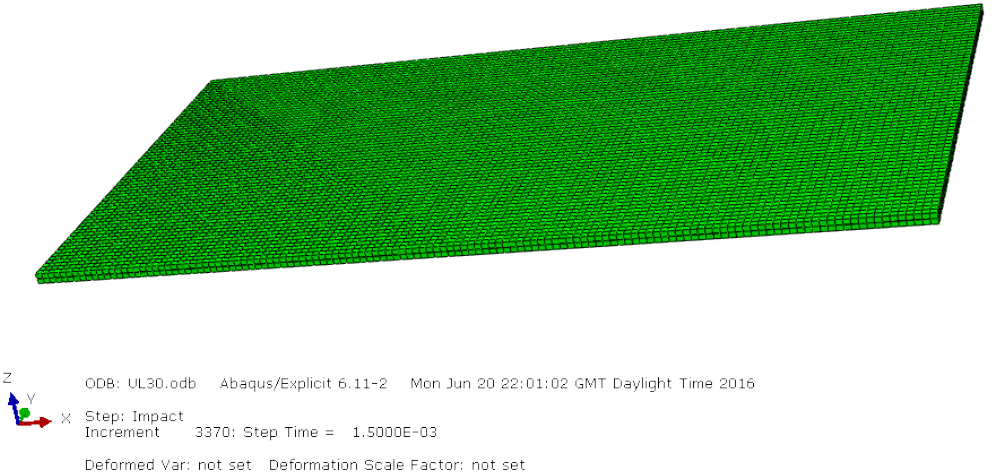

Figure 2: Mesh for 2 layers laminates for unidirectional laminates under compression.

Analysis have been carried out for $15,30,45,60$, and $75^{\circ}$ of initial fiber orientation under compression. Reaction forces variation, damage, and visco-plasticity strain are analyzed for these cases. The geometry of the models corresponds to the experiment samples with dimensions of $20 \times 10 \mathrm{~mm}^{2}$ with ply standard thickness of $0.125 \mathrm{~mm}$. A 3D 2-layer laminate of IM7-8552 is generated. The meshing of the model is shown in Fig.2. The results for this set of numerical examples are presented in subsection 4.1. Boundary condition is compressive velocity in $X$ direction so that to produce the experiment strain rate and it is fixed in another side. The boundary in $Z$ direction is symmetric. The results are presented in subsection 4.1

Another numerical model that is executed consists of \pm 45 laminate under high strain rate. A 16 ply laminate consisting of 45 degree fiber orientation in positive and negative directions consequently, is analyzed under dynamic loads in compression. The mesh for this numerical model is depicted in 3 This case is more complex in comparison to unidirectional laminates and enables a higher validation reliability. The results of the numerical analysis are compared to experiments carried out by the authors [36]. The boundary condition on both sides of the specimen is velocity which are according to applied inner and outer 


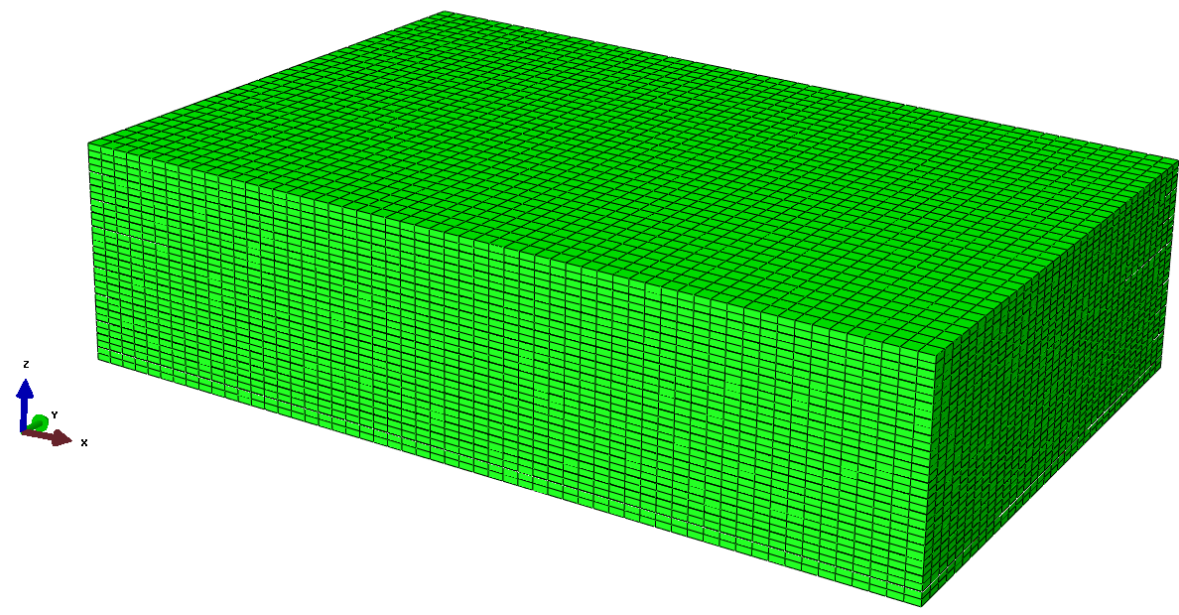

Figure 3: Mesh for 16 ply \pm 45 laminate for \pm 45 laminates under compression.

velocities that create maximum strain rate of $1300 S^{-1}$. The specimen geometry is 5 by $8 \mathrm{~mm}^{2}$. The results of this numerical example and its comparison to experimental data is provided in subsection 4.2 .

\section{Results and discussion}

\subsection{Unidirectional laminate under compression}

Figure 4 shows the reaction force versus time for the model with fiber orientation of $15^{\circ}$ direction. The Figure includes the data for quasi-static and dynamic conditions. The model for quasi-static condition considers elastic-damage behavior of the material along with bilinear plastic in shear. The dynamic model regards elastic, damage, fiber rotation, along with visco-plastic behavior and it is assumed that the strain rate is $122 S^{-1}$. As can be understood from the picture, the quasi-static model fails at $986 \mathrm{~N}$ while the dynamic one reveals failure at $1212 \mathrm{~N}$. Regarding the area of $2.5 \mathrm{~mm}^{2}$ which is under the applied load, the final stress for the first model will be $394 \mathrm{MPa}$ and strength capacity of high strain rate case will be $486 \mathrm{MPa}$. Therefore, the dynamic model is 


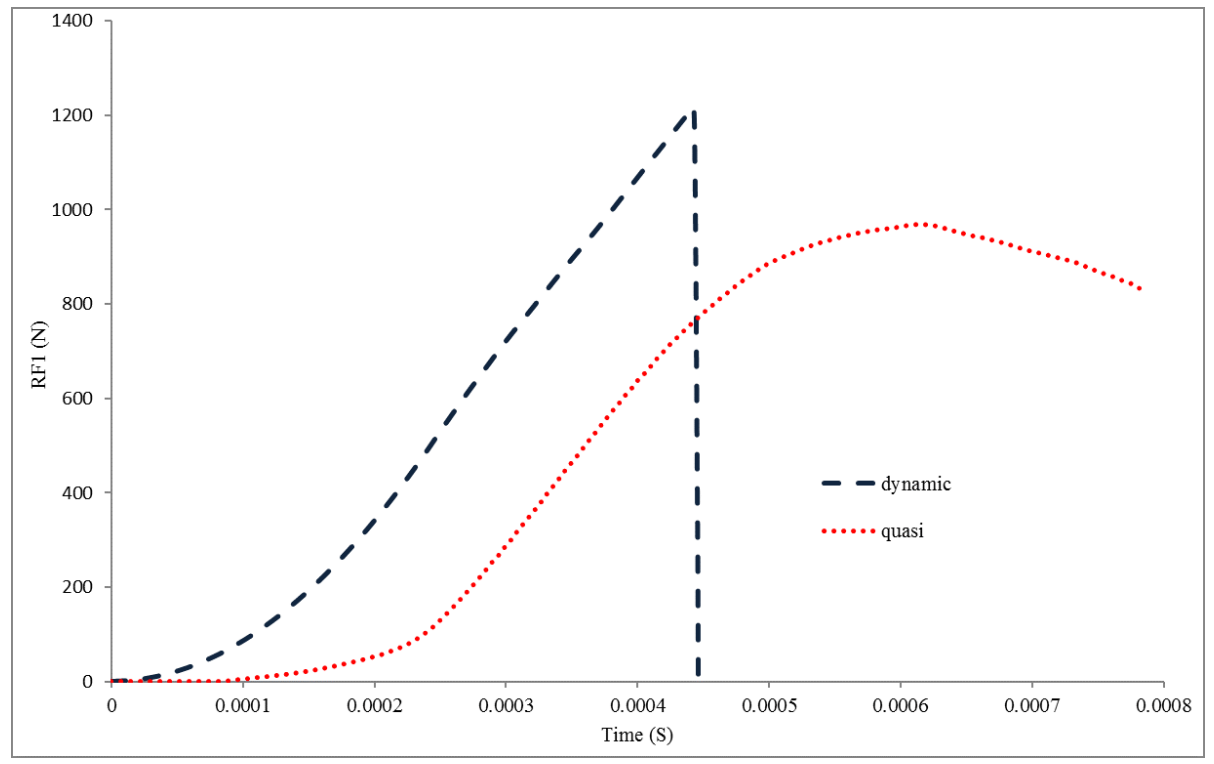

Figure 4: Comparisons of reaction forces over time for quasi-static and dynamic conditions for $15^{\circ}$ fiber laminate.

capable to capture observation reported by $[5]$ regarding the increase in failure strength due to dynamic loading. It is not only qualitatively good but also in comparison to experimental data it is accurate. According to tests conducted by [5], the final strength of IM7-8552 with fiber direction of $15^{\circ} 549 \mathrm{MPa}$ under high strain rate. Therefore, the developed model can predict the failure with a good accuracy of $11 \%$ error.

Figure 5 depicts reaction forces of the laminate under dynamic compression loads for $30^{\circ}$ model. As expected, there is an increase in the forces because of high strain rate which leads to higher failure stresses. The dynamic model fails at $1850 \mathrm{~N}$ which means $352 \mathrm{MPa}$ strength. This model is performed under the strain rate of $246 S^{-1}$. In comparison to experimental strength that is 370 $\mathrm{MPa}$, the error of the model in $30^{\circ}$ for direction of fiber is $5 \%$.

Stress-strain curve for an element in uniform area is chosen for this specimen to compare to experiment. Figure 6 illustrates the mentioned comparison which shows a good agreement between them and it can capture the nonlinearity of 


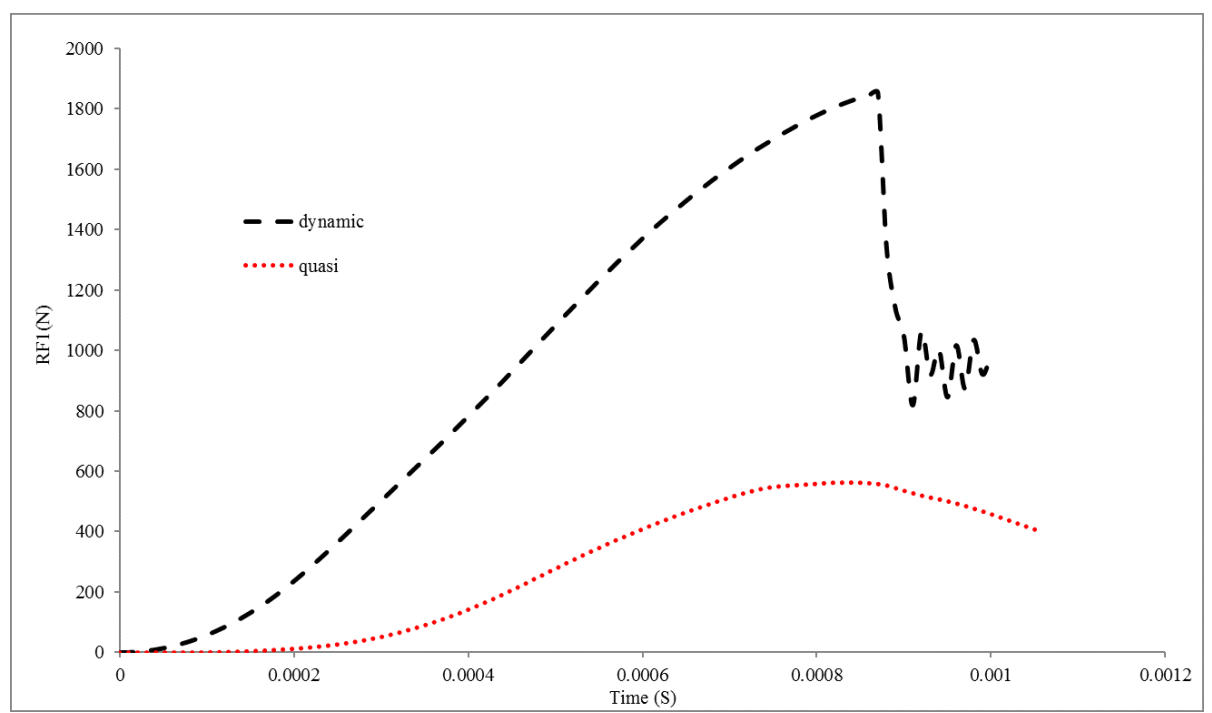

Figure 5: Comparisons of reaction forces over time for quasi-static and dynamic conditions for $30^{\circ}$ fiber laminate.

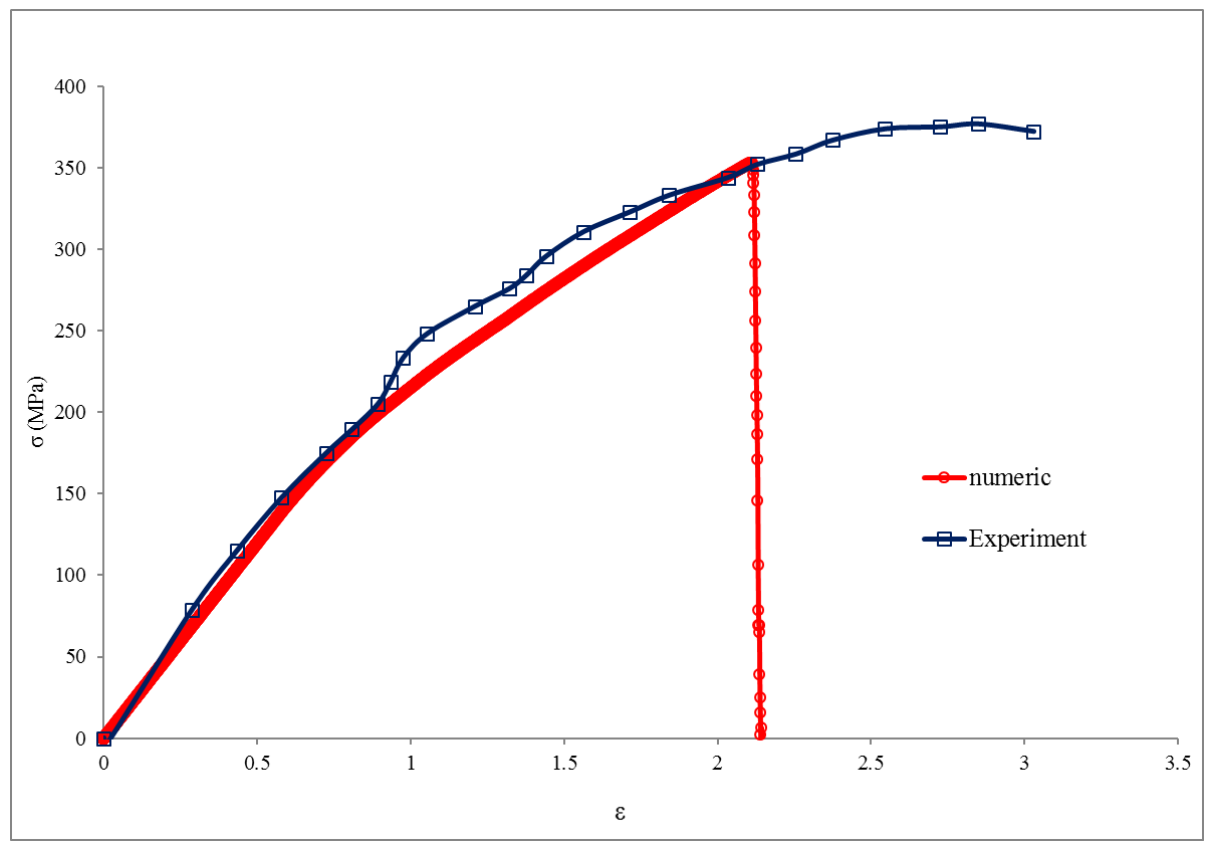

Figure 6: Stress-strain curve comparison of dynamic conditions for $30^{\circ}$ fiber laminate. 

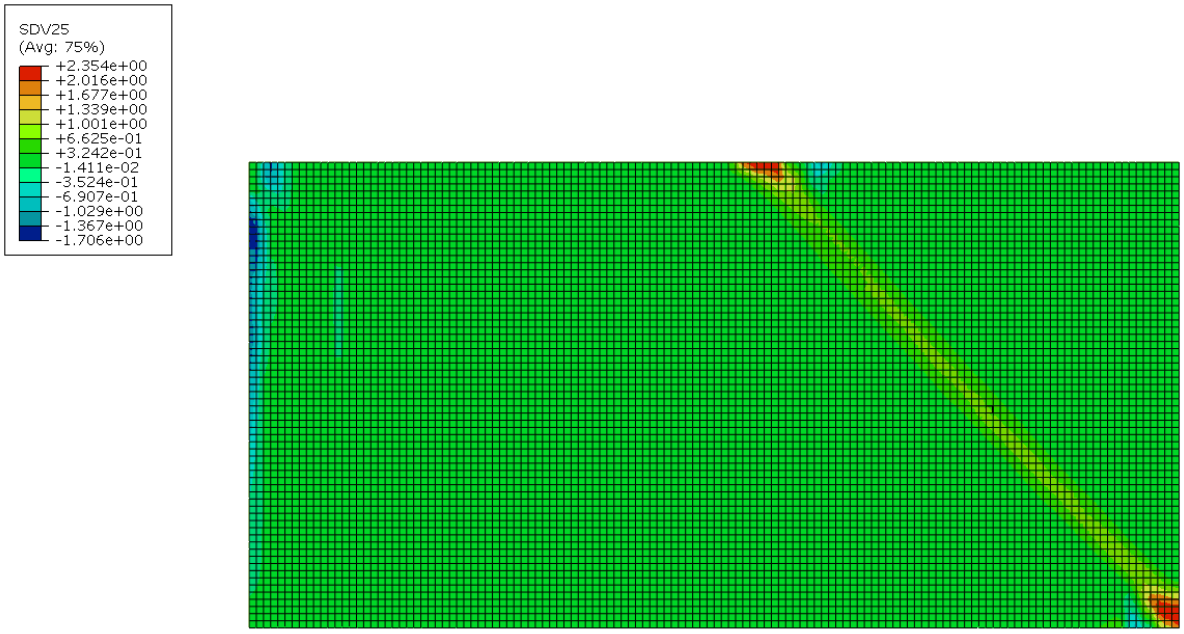

Figure 7: plastic strain in 12 direction for $30^{\circ}$ fiber laminate.

fiber rotation and visco-plasticity. Figure 7 presents the distribution of viscoplastic strain across model in the shear direction which shows a uniform viscoplastic strain.

In this model, damage initiates at one end of the specimen and evolves through the model. Figure 8 illustrates the first damage parameter, $d_{1}$, propagation during the time from $t_{1}$ to $t_{4}$. This parameter evolution is illustrated in Fig 9 . The damage parameter which represents in-plane shear is presented in Figure 10. The method of modelling damage in this research is based on continuum damage mechanics in meso-scale that has some limitation in capturing crack propagation in comparison to micro-mechanical modelling. The objective here is to find a solution to predict stress-strain curves with a reasonable computational cost. In continuum damage the material is assumed as homogenous, therefore it cannot capture material constituents with high accuracy. Besides, considering material as transversely isotropic may cause some numerical issues where it cannot differentiate between shear stresses of $s_{12}$ and $s_{21}$, therefore the damage might propagate transverse to fiber instead of aligning it.

The same trend between static and dynamic conditions is observed for $45^{\circ}$ 


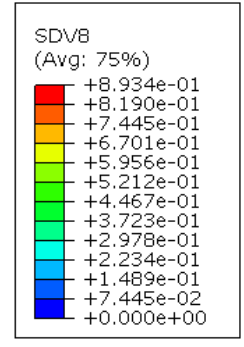

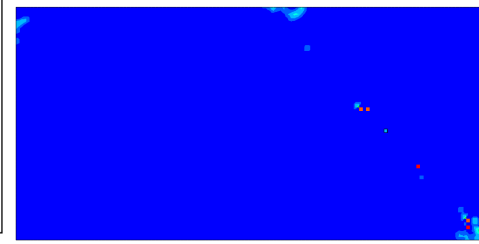

$t_{1}$

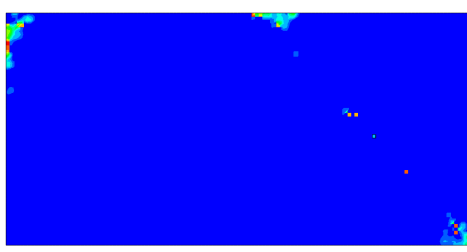

$t_{3}$

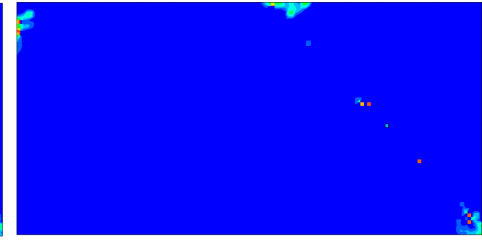

$t_{2}$

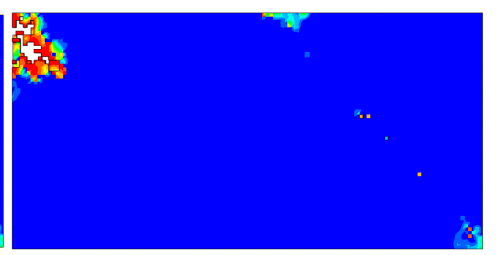

$t_{4}$

Figure 8: Evolution of $d_{1}$ damage (fiber) parameter for $30^{\circ}$ model.
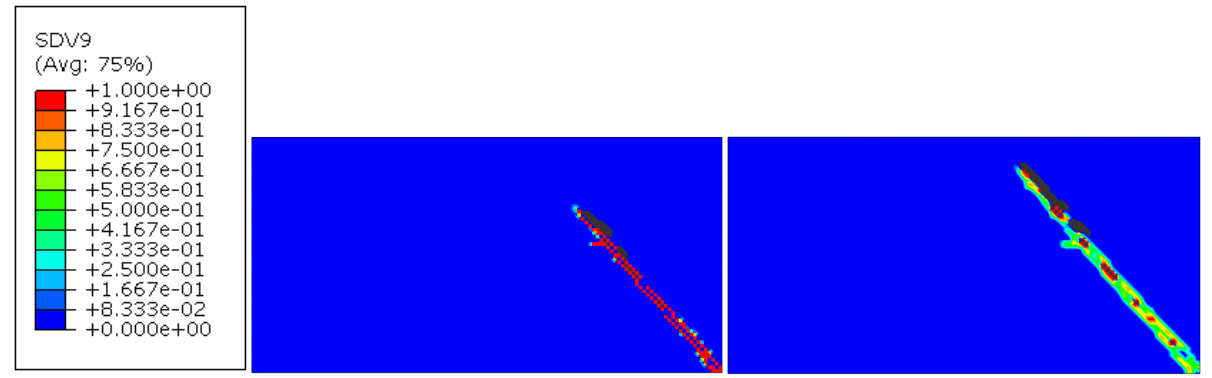

$t_{1}$

$t_{2}$

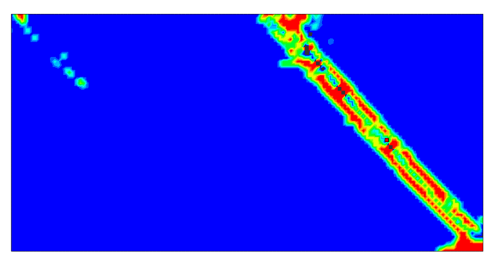

$t_{3}$

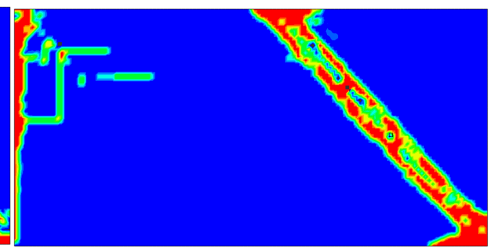

$t_{4}$

Figure 9: Evolution of $d_{2}$ damage parameter (matrix) for $30^{\circ}$ model. 

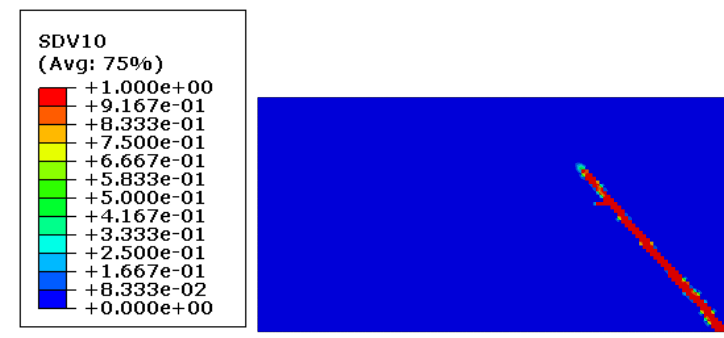

$t_{1}$

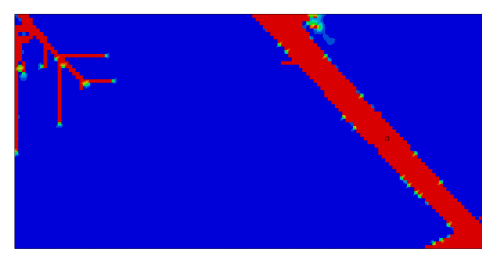

$t_{3}$

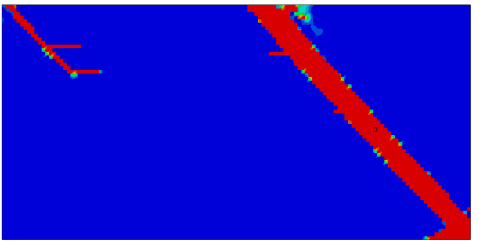

$t_{2}$

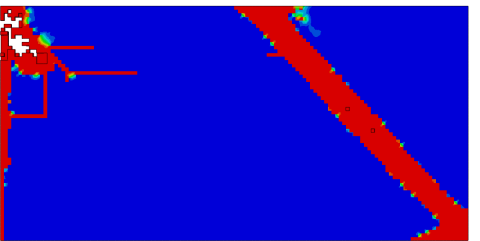

$t_{4}$

Figure 10: Evolution of $d_{6}$ damage parameter (shear) for $30^{\circ}$ model.

fibers that is shown in Fig 11. The dynamic model yields failure at $808 \mathrm{~N}$ that corresponds to $324 \mathrm{MPa}$ failure stress. In comparison to the strength observed in experiment that is $354 \mathrm{MPa}$, the model demonstrates error of $8 \%$ that is very good prediction regarding the complexity of the model and different ongoing mechanisms. From the strain point of view there is still some way to go and since the failure model triggers the damage based on final stress capacity, damage starts as soon as stress reaches close to final strength. There is no way to capture this unless by developing failure model that is based on final strain rather than final stress.

The stress-strain curve for $45^{\circ}$ fibers also expresses good accuracy that is presented in Fig. 12. The damage evolution of this model is also analyzed. The first damage parameter is depicted in Fig 13 . It also shows damage initiation in the end-load of the specimen and developing the cracks across the model. The second damage parameter and the in-plane shear are also presented in Figs. 14 and 15 , respectively. In the experimental work of [5] also reported that the 


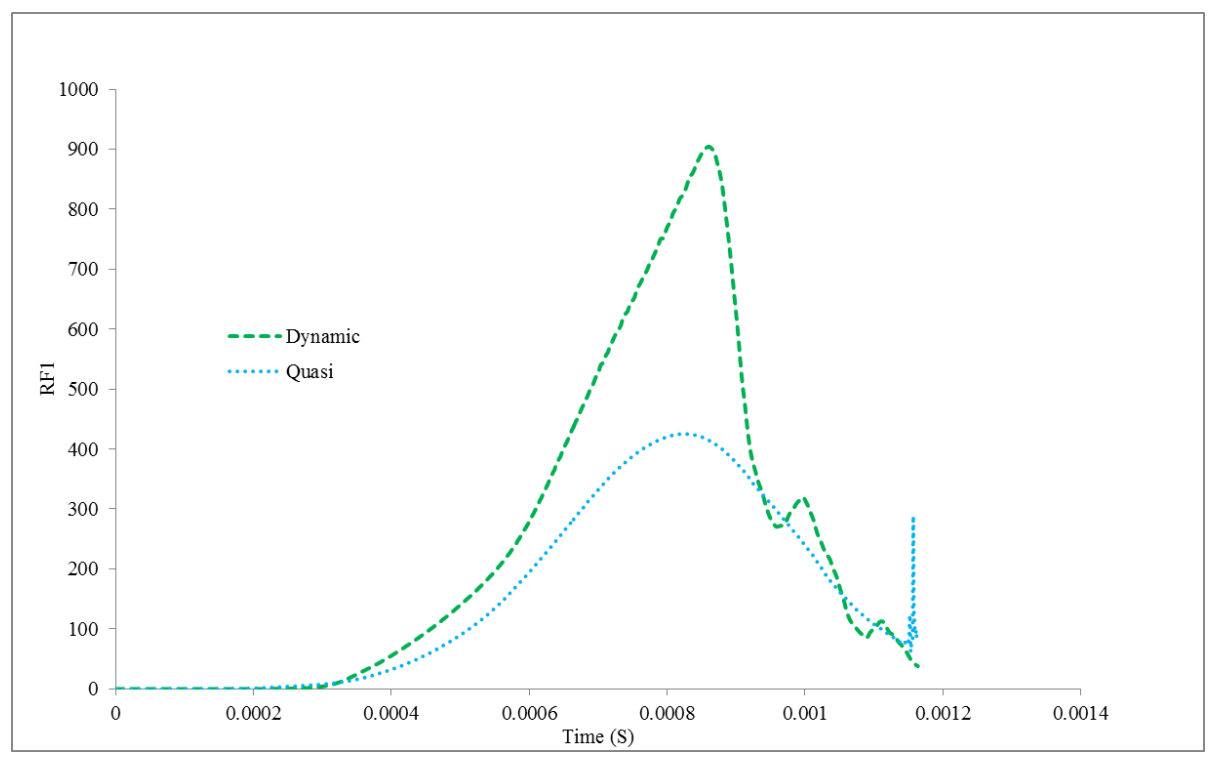

Figure 11: Comparisons of reaction forces over time for quasi-static and dynamic models for $45^{\circ}$ fiber laminate.

damage grows from end-load of the specimen. In addition the X-ray image of the failed specimen from that, has a correlation with damage parameters that implies ability of the model in capturing the failure plane. This comparison is shown in Fig. 17.

The visco-plastic strain in shear direction for this model is also presented $\mathrm{n}$ Fig. 16 that in comparison to $30^{\circ}$ model has higher progress in the loading end of the specimen. These strains are also higher in comparison to visco-plastic in $30^{\circ}$ model.

The model has a good ability to predict the final strength of the laminates for higher fiber orientation as well. Figure 18 illustrates the forces in $60^{\circ}$ model. It fails at $913 \mathrm{~N}$. The comparison between stress-strain curves from the model and experiment for $60^{\circ}$ is depicted in 19 which shows an excellent agreement with experiments. The curve predicts the response very well in linear and nonlinear parts and damage triggers at the right strength.

Table 6 shows the final strength predicted by the model and the ones achieved 


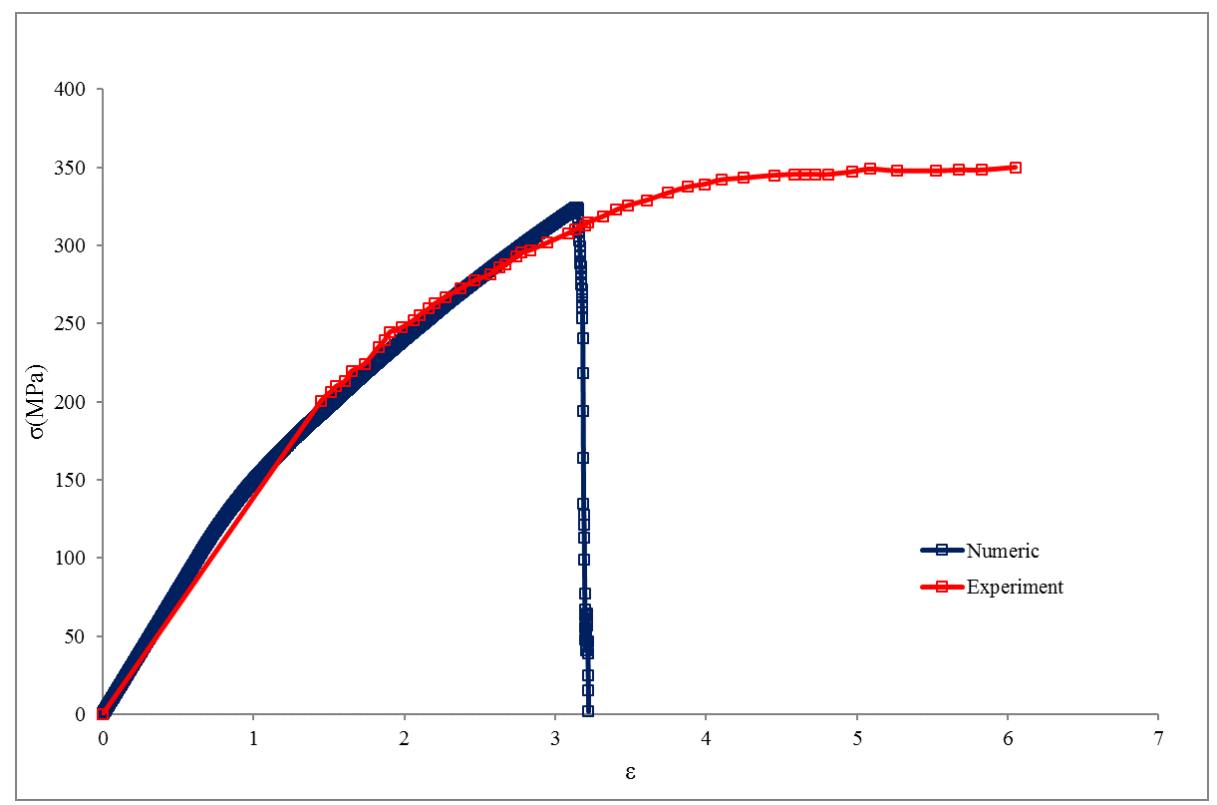

Figure 12: Stress-strain curve comparison of dynamic conditions for $45^{\circ}$ fiber laminate.
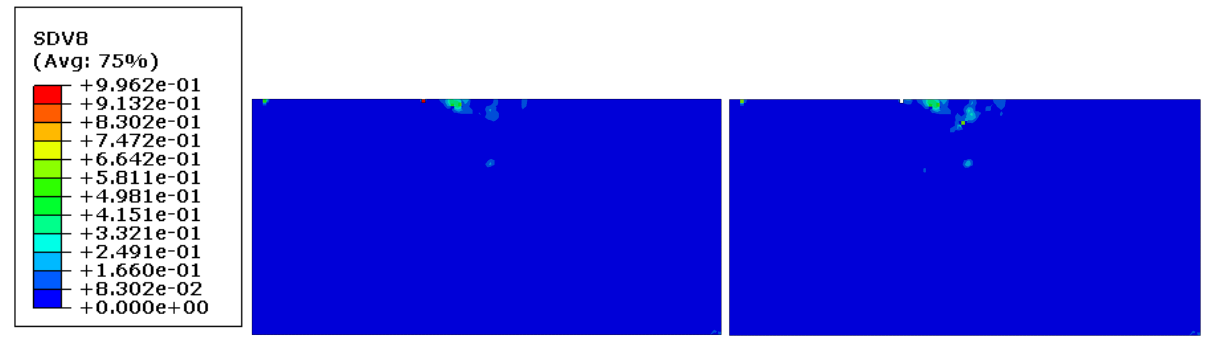

$t_{1}$

$t_{2}$
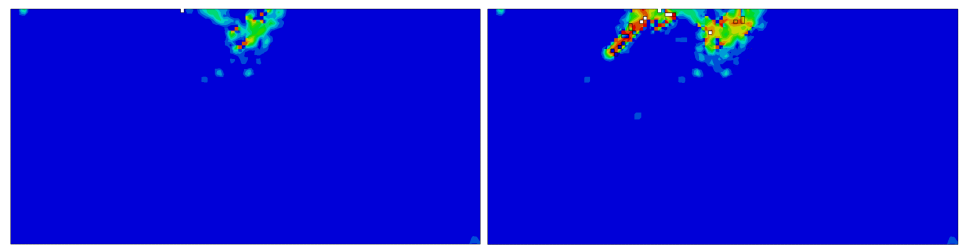

$t_{3}$

$t_{4}$

Figure 13: Evolution of $d_{1}$ damage parameter for $45^{\circ}$ model. 

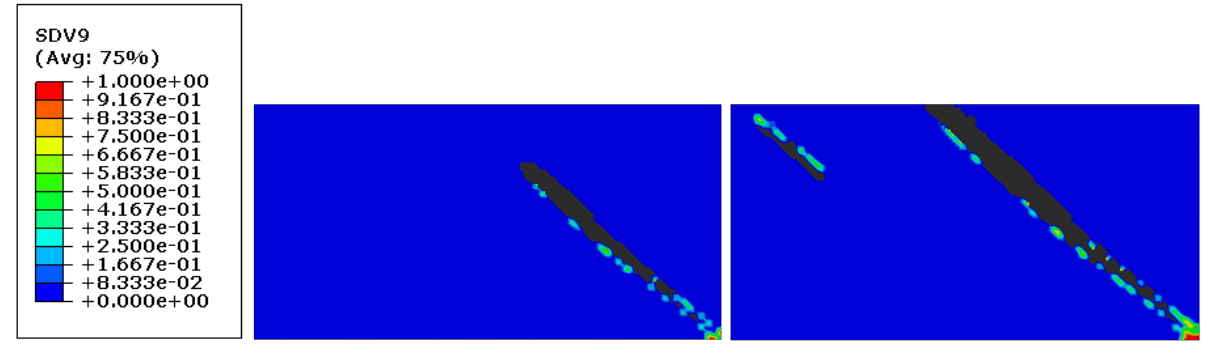

$t_{1}$

$t_{2}$

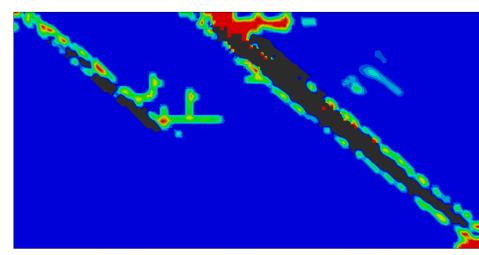

$t_{3}$

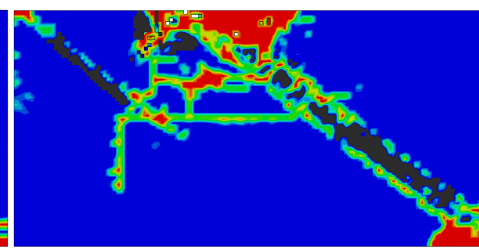

$t_{4}$

Figure 14: Evolution of $d_{2}$ damage parameter for $45^{\circ}$ model.

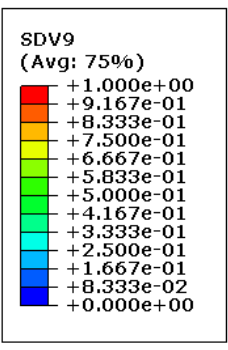

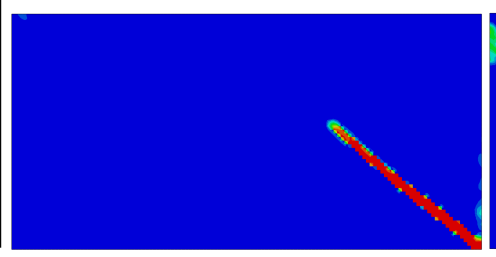

$t_{1}$

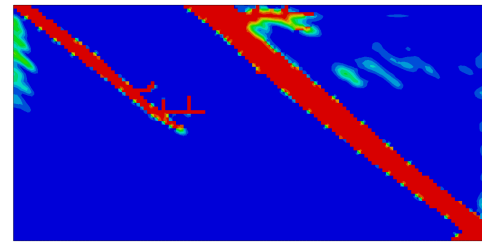

$t_{3}$

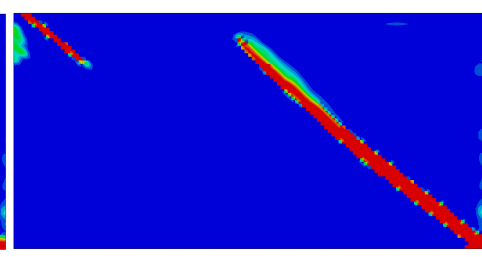

$t_{2}$

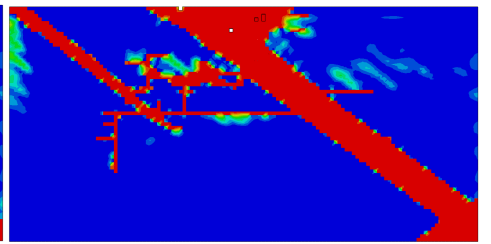

$t_{4}$

Figure 15: Evolution of $d_{6}$ damage parameter for $45^{\circ}$ model. 

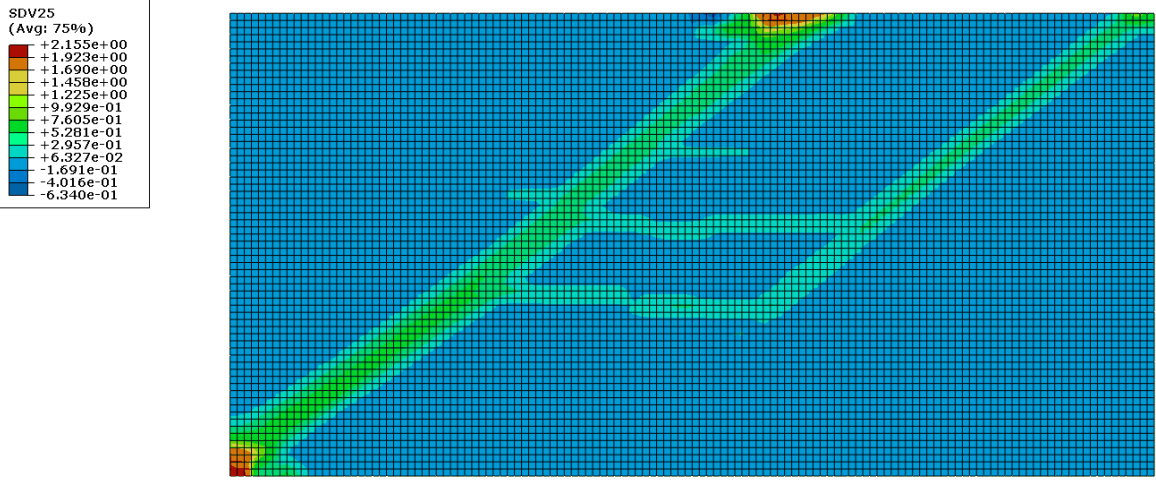

Figure 16: plastic strain in 12 direction for $45^{\circ}$ fiber laminate.

by experiments that illustrates capability of the numerical model to predict final strength under dynamic loads. The model with direction of fiber in $60^{\circ}$ has the best accuracy among all the directions.

Table 6: Final strength for different fiber orientation under dynamic loads.

\begin{tabular}{ccccc}
\hline Orientation $\left(^{\circ}\right)$ & Strain rate $\left(S^{-1}\right)$ & Experiment $(\mathrm{MPa})$ & Numeric $(\mathrm{MPa})$ & Error \\
\hline 15 & 122 & 549 & 486 & $11 \%$ \\
30 & 246 & 370 & 352 & $5 \%$ \\
45 & 280 & 354 & 324 & $8 \%$ \\
60 & 331 & 365 & 366 & $0 \%$ \\
75 & 305 & 371 & 350 & $9 \%$ \\
\hline
\end{tabular}

\section{2. \pm 45 laminate under compression}

The laminate final failure capacity in finite element model is $2582 \mathrm{~N}$ while in the experimental data it is $2905 \mathrm{~N}$ which means $11 \%$ of error. The reaction force versus time diagram is presented in Figure 20. It should be mentioned that the strain rate is high and the visco-elastic model that is used for modeling is mainly recommended for medium and low rate, therefore there is some room for 


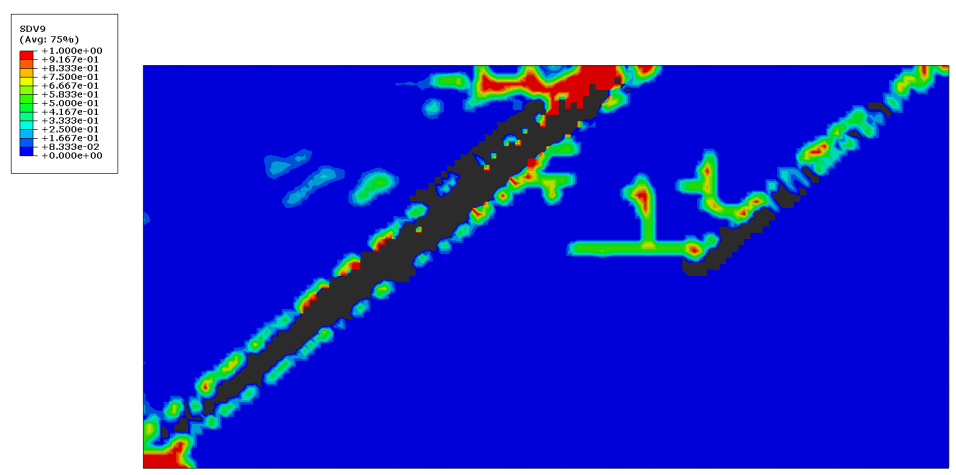

(a) $d_{2}$

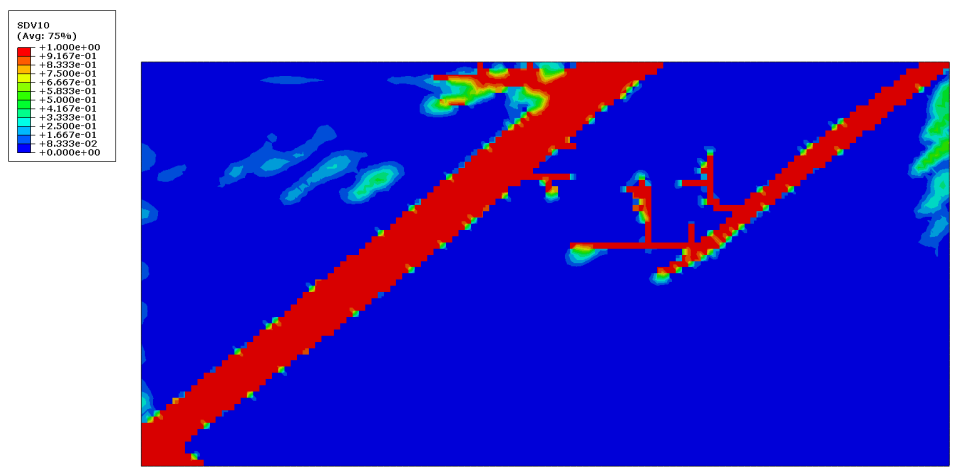

(b) $d_{6}$

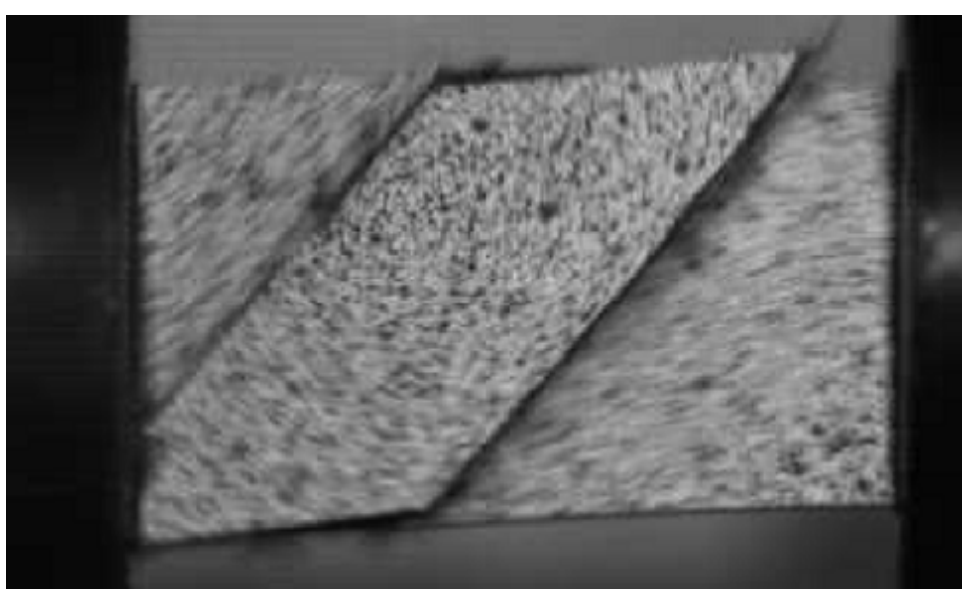

(c) Experiment [5

Figure 17: Comparison of failed specimen in experiment and numeric model for $45^{\circ}$ model. 


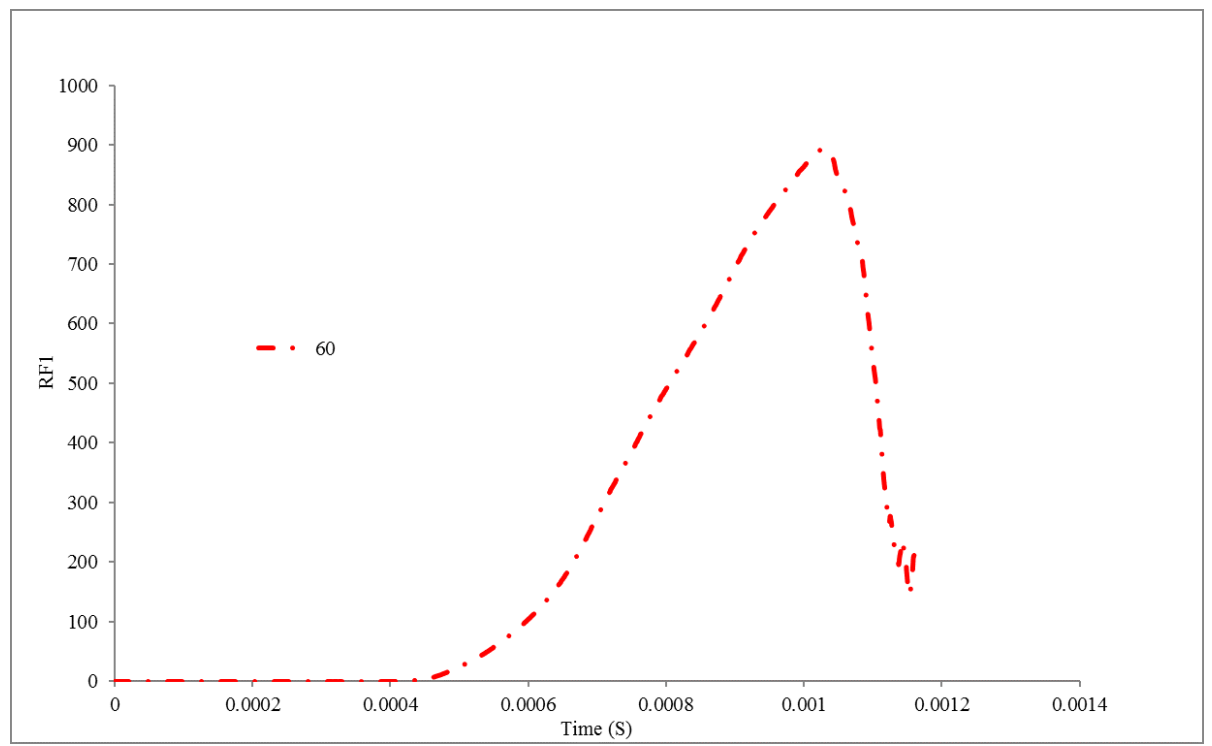

Figure 18: Reaction forces versus time under dynamic conditions for $60^{\circ}$ laminate.

more improvement on that model. The dominant damage mode is expected to be shear which is represented by damage variable of $d_{6}$. This can be captured by model that is illustrated in Figure 21. It is also compared with samples picture after test which shows the fracture angle corresponds to maximum damage parameter. Therefore, the model is capable of capturing the fracture plane. The plastic shear strain is calculated in the model and illustrated in Figure 22.

\section{Conclusion}

A phenomenological constitutive model is developed to analyze composites under dynamic conditions including low and medium strain rates. The model is capable of capturing nonlinearities due to visco-plasticity. It can model the material response very well and predicts final failure of the laminates in a very difficult cases to predict with good accuracy. It showed that the strain rate increases the failure capacity of the laminate. The model is capable to find the damage initiation point and the dominant failure mode along with fracture 


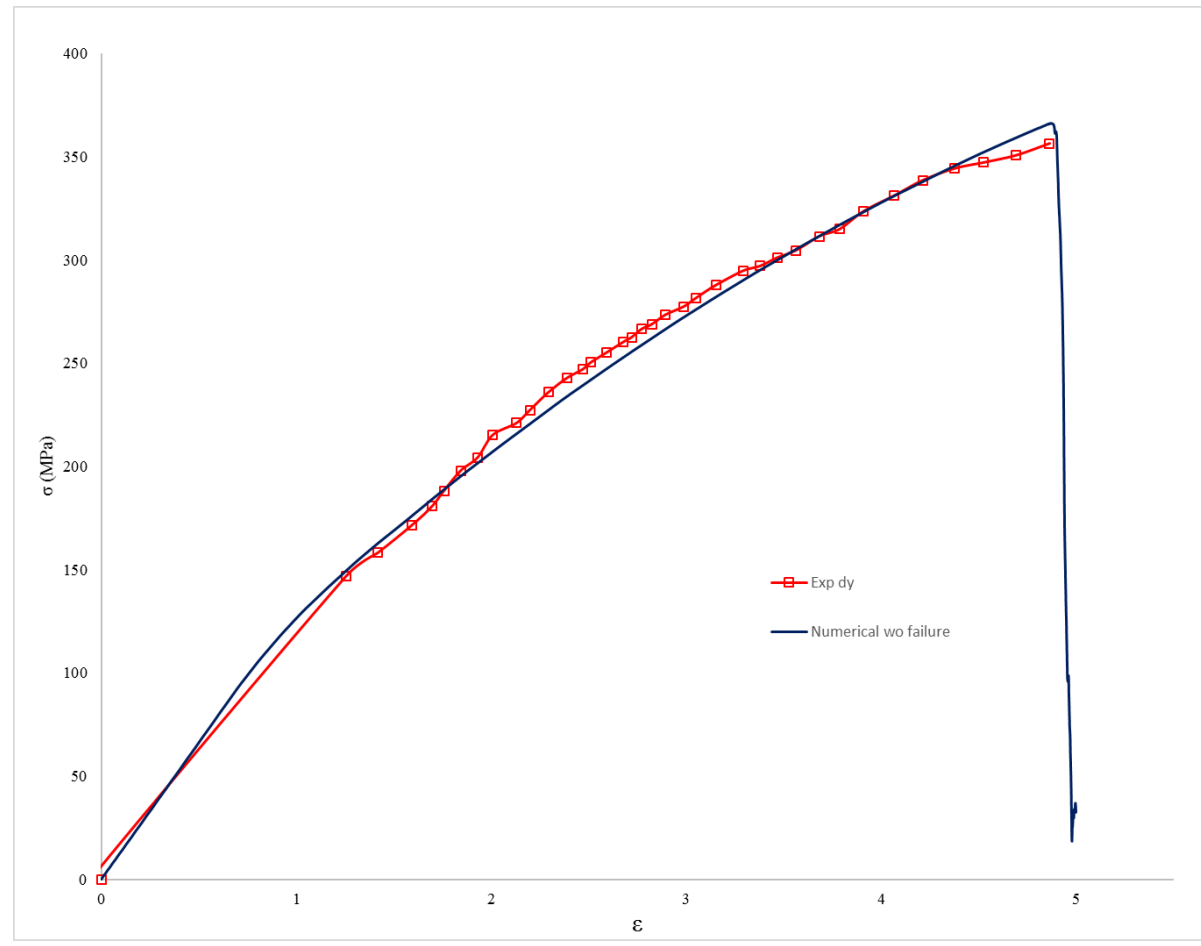

Figure 19: Stress-strain curve comparison of dynamic conditions for $60^{\circ}$ fiber laminate.

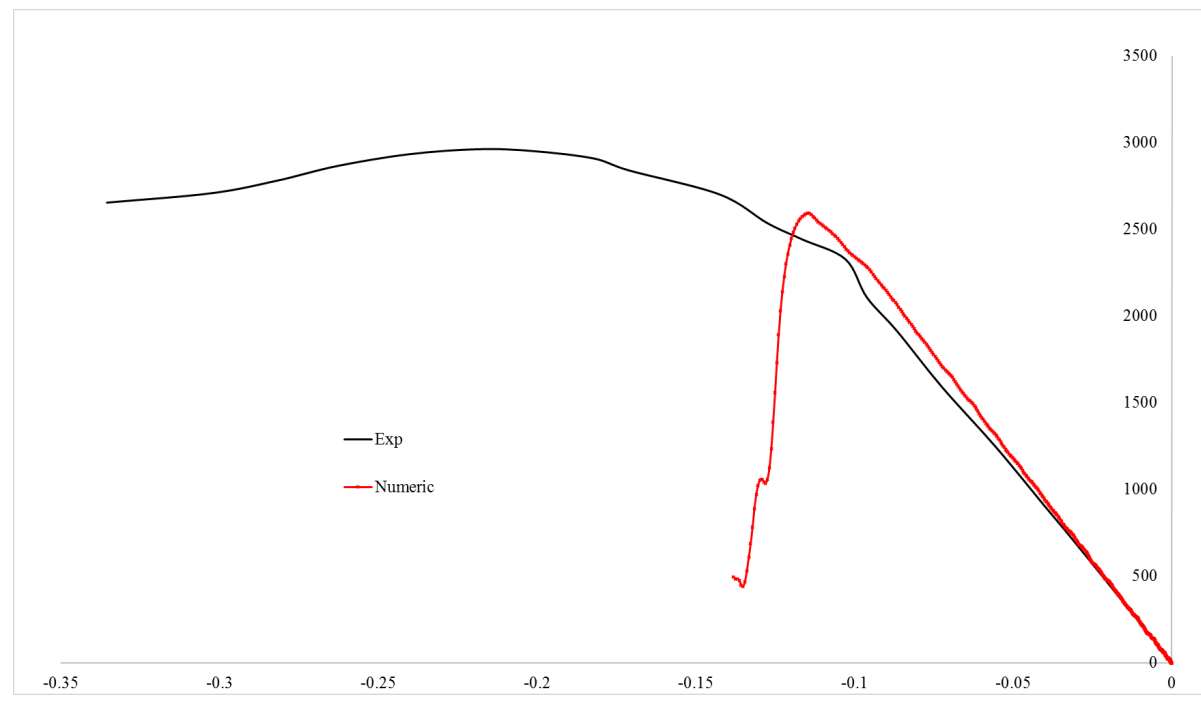

Figure 20: Reaction forces versus time under dynamic conditions for $\pm 45^{\circ}$ laminate. 

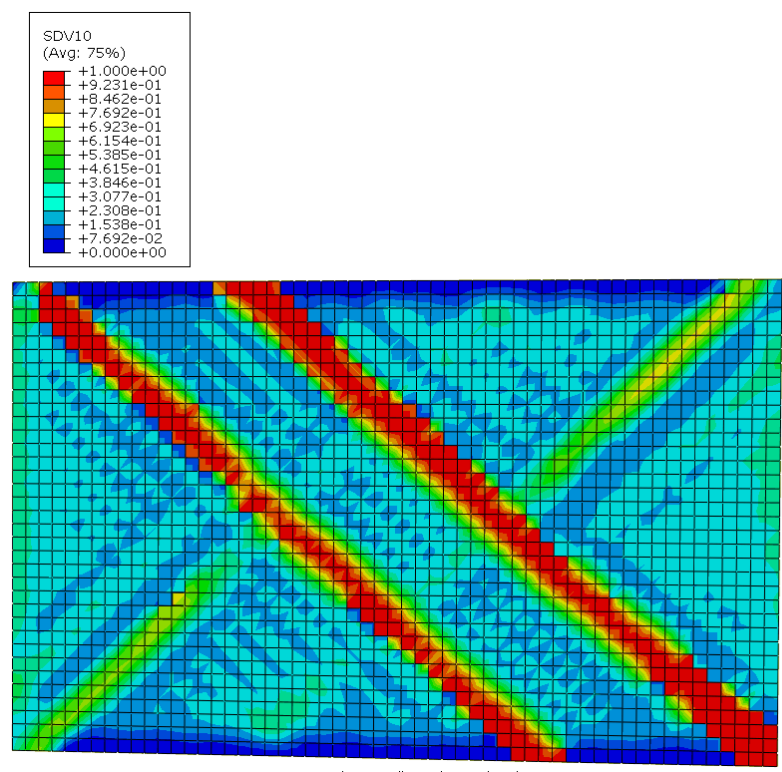

(a) Finite element model

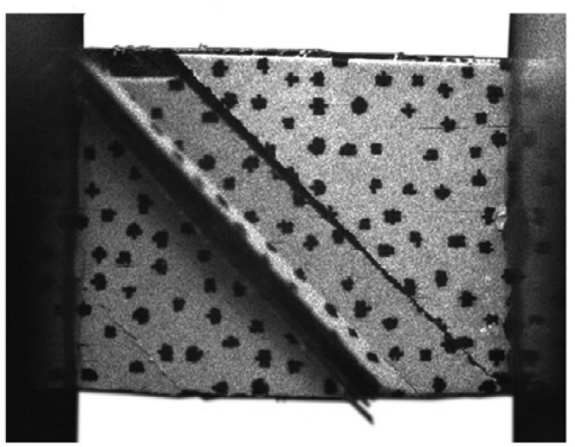

(b) Test Sample

Figure 21: Comparison of shear damage variable in numerical model and fracture plane in test sample for $\pm 45^{\circ}$ laminate in compression 


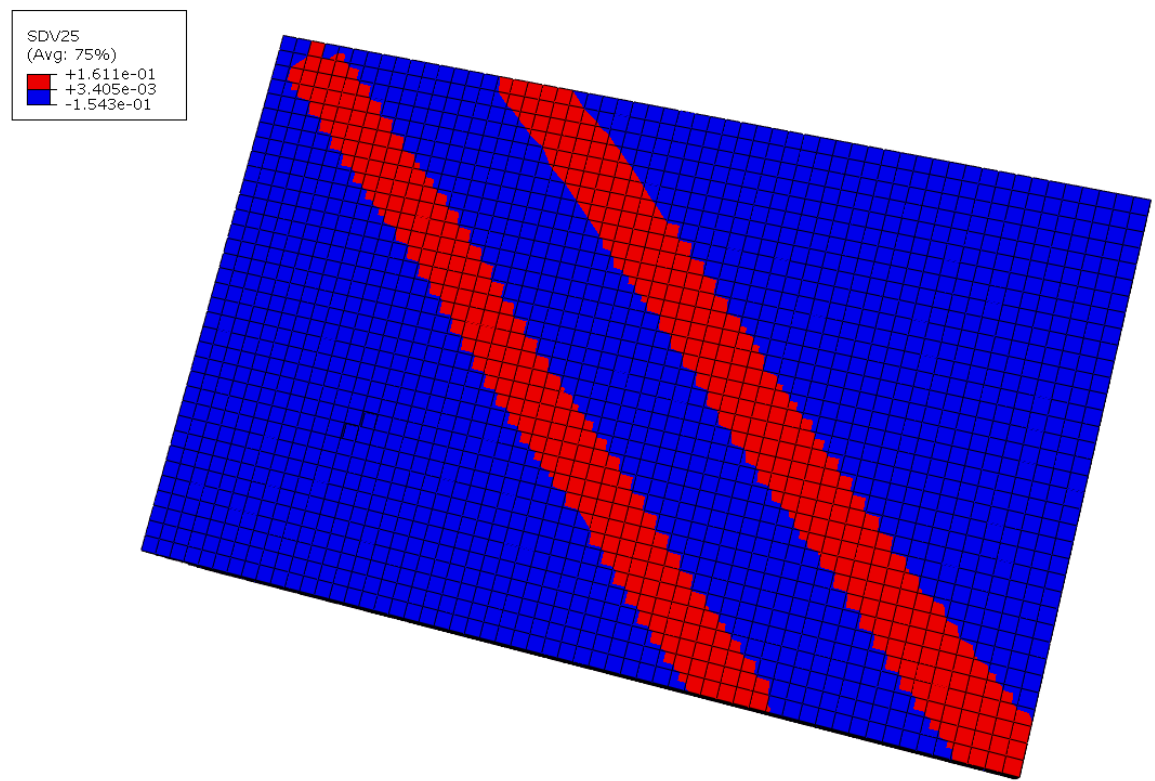

Figure 22: Plastic shear strain for $\pm 45^{\circ}$ laminate.

plane. It is recommended to perform more evaluation to be done for failure models based on strain rather than stress.

\section{Acknowledgments}

The financial support of the FCT under individual research scholarship SFRH/BD/103436/2014 is acknowledged. In addition, the support of Department of Engineering Science at University of Oxford is appreciated. The authors are also grateful of useful discussions with Dr. Borja Erice from Norwegian University of Science and Technology.

\section{References}

[1] K. Yoon, C. Sun, Characterization of elastic-viscoplastic properties of an as4/peek thermoplastic composite, Journal of Composite Materials 25 (10) (1991) 1277-1296. 
[2] J. M. Lifshitz, Impact strength of angle ply fiber reinforced materials, Journal of Composite Materials 10 (1) (1976) 92-101.

[3] I. Daniel, Fracture of hybrid composite materials under dynamic loading, in: Composites Design: Proceedings of the Ninth International Conference on Composite Materials (ICCM/9), Madrid, 12-16 July, 1993. volume 4, Vol. 4, Woodhead Publishing, 1993.

[4] B. M. Powers, J. R. Vinson, M. Wardle, B. Scott, High strain rate effects on two as 4 graphite fiber polymer matrix composites.

[5] H. Korber, J. Xavier, P. P. Camanho, High strain rate characterisation of unidirectional carbon-epoxy im7-8552 in transverse compression and in-plane shear using digital image correlation, Mechanics of Materials 42 (2010) 1004-1019.

[6] H. Korber, Mechanical response of advanced composites under high strain rates, Ph.D. thesis, University of Porto, 2010.

[7] R. Gerlach, C. R. Siviour, J. Wiegand, N. Petrinic, The strain rate dependent material behavior of s-gfrp extracted from glare, Mechanics of Advanced Materials and Structures 20 (7) (2013) 505514. arXiv:https://doi.org/10.1080/15376494.2011.627646, doi: $10.1080 / 15376494.2011 .627646$.

URL https://doi.org/10.1080/15376494.2011.627646

[8] Y. Zhou, Y. Wang, S. Jeelani, Y. Xia, Experimental study on tensile behavior of carbon fiber and carbon fiber reinforced aluminum at different strain rate, Applied Composite Materials 14 (1) (2007) 17-31.

[9] A. Gilat, R. K. Goldberg, G. D. Roberts, Experimental study of strainrate-dependent behavior of carbon/epoxy composite, Composites Science and Technology 62 (10-11) (2002) 1469-1476. 
[10] A. Gilat, R. K. Goldberg, G. D. Roberts, Strain rate sensitivity of epoxy resin in tensile and shear loading, Journal of Aerospace Engineering 20 (2) (2007) 75-89.

[11] R. Gerlach, C. R. Siviour, N. Petrinic, J. Wiegand, Experimental characterisation and constitutive modelling of rtm-6 resin under impact loading, Polymer 49 (11) (2008) 2728-2737.

[12] M. Vogler, H. Koerber, P. Kuhn, R. Rolfes, P. Camanho, Constitutive modeling and experimental characterization of the non-linear stress-strain behavior of unidirectional carbon-epoxy under high strain rates, in: Proceedings of the 20th International Conference on Composite Materials (ICCM/20), Copenhagen, 19-24 July, 2005.

[13] M. Vogler, R. Rolfes, P. Camanho, Modeling the inelastic deformation and fracture of polymer composites-part i: plasticity model, Mechanics of Materials 59 (2013) 50-64.

[14] B. Erice, D. Thomson, S. A. Ponnusami, M. V. Pathan, N. Petrinic, On the rate-dependent plasticity modelling of unidirectional fibre-reinforced polymeric matrix composites, in: European Physical Journal Web of Conferences, Vol. 183, 2018.

[15] J. Aboudi, A continuum theory for fiber-reinforced elastic-viscoplastic composites, International Journal of Engineering Science 20 (5) (1982) 605-621.

[16] A. Saleeb, T. Wilt, Analysis of the anisotropic viscoplastic-damage response of composite laminatescontinuum basis and computational algorithms, International journal for numerical methods in engineering 36 (10) (1993) $1629-1660$.

[17] C. Lissenden, C. Herakovich, Numerical modelling of damage development and viscoplasticity in metal matrix composites, Computer Methods in Applied Mechanics and Engineering 126 (3) (1995) 289-303. 
[18] D. Vasiukov, S. Panier, A. Hachemi, Non-linear material modeling of fiber-reinforced polymers based on coupled viscoelasticity-viscoplasticity with anisotropic continuous damage mechanics, Composite Structures 132 (2015) 527-535.

[19] P. Perzyna, Fundamental problems in viscoplasticity, in: Advances in applied mechanics, Vol. 9, Elsevier, 1966, pp. 243-377.

[20] P. Kuhn, G. Catalanotti, J. Xavier, P. Camanho, H. Koerber, Fracture toughness and crack resistance curves for fiber compressive failure mode in polymer composites under high rate loading, Composite Structures 182 (2017) 164-175.

[21] P. Kuhn, G. Catalonotti, J. Xavier, R. Cidade, H. Koerber, P.P. Camanho, Measurement of fracture toughness for fiber compressive failure mode of fiber composites under high rate loading (2016).

[22] D. Allen, R. Jones, J. Boyd, Micromechanical analysis of a continuous fiber metal matrix composite including the effects of matrix viscoplasticity and evolving damage, Journal of the Mechanics and Physics of Solids 42 (3) (1994) 505-529.

[23] N. Ohno, X. Wu, T. Matsuda, Homogenized properties of elasticviscoplastic composites with periodic internal structures, International Journal of Mechanical Sciences 42 (8) (2000) 1519-1536.

[24] M. Megnis, J. Varna, Micromechanics based modeling of nonlinear viscoplastic response of unidirectional composite, Composites Science and Technology 63 (1) (2003) 19-31.

[25] O. Pierard, J. LLorca, J. Segurado, I. Doghri, Micromechanics of particlereinforced elasto-viscoplastic composites: finite element simulations versus affine homogenization, International Journal of Plasticity 23 (6) (2007) 1041-1060. 
[26] M. Pathan, S. Patsias, J. Rongong, V. Tagarielli, Measurements and predictions of the viscoelastic properties of a composite lamina and their sensitivity to temperature and frequency, Composites Science and Technology.

[27] M. Al-Haik, H. Garmestani, A. Savran, Explicit and implicit viscoplastic models for polymeric composite, International Journal of Plasticity 20 (10) (2004) 1875-1907.

[28] S. Eskandari, F. M. A. Pires, P. P. Camanho, A. T. Marques, Intralaminar damage in polymer composites in the presence of finite fiber rotation: Part i constitutive model, Composite Structures 151 (2016) 114126.

[29] S. Eskandari, F. M. A. Pires, P. P. Camanho, A. T. Marques, Intralaminar damage in polymer composites in the presence of finite fiber rotation: Part ii numerical analysis and validation, Composite Structures 151 (2016) 127141.

[30] S. Eskandari, F. M. A. Pires, P. P. Camanho, A. T. Marques, Analysis of transverse compression and in-plane shear in unidirectional composites by a progressive damage model in presence of fiber rotation (2016).

[31] S. T. Pinho, C.G. Davila, P.P. Camanho, L. Iannuccic, P. Robinson, Failure models and criteria for frp under in-plane or threedimensional stress states including shear non-linearity, Tech. Rep. NASA/TM-2005-213530, NASA, Langley Research Center, Hampton, VA (2005).

[32] P. Maimi, P. P. Camanho, J. A. Mayugo, C. G. Davila, A continuum damage model for composite laminates: Part i constitutive model, Mechanics of Materials 39 (2007) 897908.

[33] P. Maimi, P. P. Camanho, J. A. Mayugo, C. G. Davila, A continuum damage model for composite laminates: Part i constitutive model, Mechanics of Materials 39 (2007) 903919. 
[34] P. Camanho, P. Maimi, C. Davila, Prediction of size effects in notched laminates using continuum damage mechanics, Composites Science and Technology 67 (2007) 27152727.

[35] P. Camanho, C. Davila, S. T. Pinho, L. Iannuccic, P. Robinson, Prediction of in situ strengths and matrix cracking in composites under transverse tension and in-plane shear, Composites: Part A 37 (2006) 165176.

[36] H. Cui, D. Thomson, A. Pellegrino, J. Wiegand, N. Petrinic, Effect of strain rate and fibre rotation on the in-plane shear response of 45 laminates in tension and compression tests, Composites Science and Technology 135 (2016) 106-115. 
2019-03-19

\section{Analyzing the failure and damage of FRP composite laminates under high strain rates considering visco-plasticity}

\section{Eskandari, Sina}

Elsevier

Escandari S, Andrade Pires FM, Camanho PP, et al., Analyzing the failure and damage of FRP composite laminates under high strain rates considering visco-plasticity. Engineering Failure Analysis, Volume 101, July 2019, pp. 257-273

https://doi.org/10.1016/j.engfailanal.2019.03.008

Downloaded from Cranfield Library Services E-Repository 